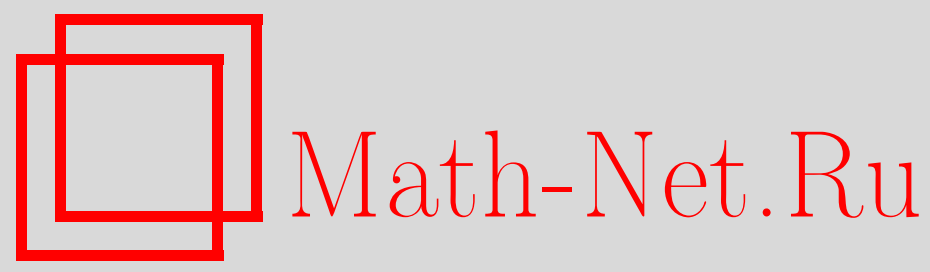

В. Б. Шерстюков, Об одной задаче Леонтьева и представляющих системах экспонент, Матем. заметки, 2003, том 74, выпуск 2, 301-313

DOI: https://doi.org/10.4213/mzm264

Использование Общероссийского математического портала Math-Net.Ru подразумевает, что вы прочитали и согласны с пользовательским соглашением http://www . mathnet.ru/rus/agreement

Параметры загрузки:

IP: 54.197 .130 .99

26 апреля 2023 г., 14:21:01

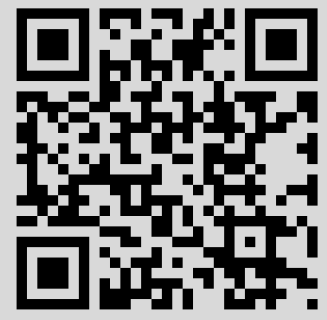




\title{
ОБ ОДНОЙ ЗАДАЧЕ ЛЕОНТЬЕВА И ПРЕДСТАВЛЯЮЩИХ СИСТЕМАХ ЭКСПОНЕНТ
}

\author{
В.Б. Шерстюков
}

Изучается вопрос о наличии вполне регулярного роста у целой функции экспоненциального типа, производная которой достаточно быстро растет на нулевом множестве самой функции. В частности, для функции с тригонометрически выпуклым (или положительньмм) нижним индикатором получено решение одной известной задачи Леонтьева. В качестве приложения дается уточнение некоторых известных ранее результатов о характеризации показателей представляющих систем экспонент.

Библиография: 13 названий.

1. Введение. Формулировка основных результатов. Изучая вопросы, связанные с представлением функций рядами экспонент, Леонтьев в работе [1] поставил следующую задачу. Пусть $\mathscr{L}(\lambda)$ - целая функция экспоненциального типа (ц.ф.э.т.) с последовательностью простых (всех) нулей $\Lambda=\left(\lambda_{k}\right)_{k=1}^{\infty}$ и индикатором $h(\mathscr{L} ; \theta)$, удовлетворяющая условию

$$
\lim _{k \rightarrow \infty}\left\{\frac{1}{\left|\lambda_{k}\right|} \ln \left|\mathscr{L}^{\prime}\left(\lambda_{k}\right)\right|-h\left(\mathscr{L} ; \arg \lambda_{k}\right)\right\}=0 .
$$

Требуется выяснить, является ли $\mathscr{L}$ функцией вполне регулярного роста ${ }^{1}$ (в.р.р.).

Указанная задача исследовалась Леонтьевым, Мельником, Братищевым как в приведенной вьше, так и в более общей постановке (для целой функции с индикатором $\mathscr{H}(\mathscr{L} ; \theta)$ при уточненном порядке $\rho(r) \rightarrow \rho \in[0, \infty))$. Она имеет положительное решение при $\rho \in[0,1 / 2)$; тот же результат получен [3] для $\rho \in[1 / 2,1)$ при дополнительных предположениях

$$
\min _{\theta} \mathscr{H}(\mathscr{L}, \theta)>0 \text { и } \varlimsup_{r \rightarrow \infty} \frac{\ln \min _{|\lambda|=r}|\mathscr{L}(\lambda)|}{\ln r}=+\infty .
$$

Следует отметить, что при $\rho(r) \equiv \rho$ из теоремы 1 работы Мельника [4] и теоремы 3 обзорной статьи Коробейника [5, c. 106-107] следует справедливость цитированных результатов Братищева уже при любом $\rho>0$. Если условие положительности индикатора нарушено, то при $\rho \geqslant 1 / 2$ соотношение (1) или его естественное обобщение (в случае $\rho(r) \not \equiv 1)$ не влекут, вообще говоря, полной регулярности роста функции. В частности,

\footnotetext{
${ }^{1}$ Используемые нами без пояснений сведения из теории целых функций можно найти, например, в [2].
} 
в [3] фактически построен пример четной ц.ф.э.т. с вещественньпи нулями, удовлетворяющей условию (1), но не имеющей в.p.p.

Обозначим через $L$ класс функщий, удовлетворяюших условию “классической" задачи Леонтьева. Вопрос о полной регулярности роста функции $\mathscr{L} \in L$, индикаторная диаграмма которой имеет внутренние точки, в общей ситуации остается открытьп. Результаты Мельника позволяют дать положительньй ответ на него в случае наличия особой симметрии в расположении корней $\mathscr{L}$ или определенной оценки снизу роста $|\mathscr{L}(\lambda)|$ на некоторой системе расширяющихся до бесконечности окружностей $|\lambda|=R_{n}$ (например, $\min _{|\lambda|=R_{n}}|\mathscr{L}(\lambda)|>R_{n}^{-p}$ при каком-либо $\left.p>0\right)$. Дополнительные сведения из истории вопроса и точные формулировки соответствующих результатов можно найти в диссертации [6].

В связи с изложенным вьше возникает ряд естественных вопросов. В настоящей статье наряду с задачей Леонтьева изучаются два из них. Во-первых, будет ли иметь вполне регулярньй рост ц.ф.э.т. $\mathscr{L}$ с простьпи нулями $\left(\lambda_{k}\right)_{k=1}^{\infty}$ и индикатором $h(\mathscr{L} ; \theta)>0$, удовлетворяющая более слабому, чем (1), условию: существует $\gamma \in(0,1)$ такая, что

$$
\varliminf_{k \rightarrow \infty}\left\{\frac{1}{\left|\lambda_{k}\right|} \ln \left|\mathscr{L}^{\prime}\left(\lambda_{k}\right)\right|-\gamma h\left(\mathscr{L}, \arg \lambda_{k}\right)\right\} \geqslant 0
$$

(такие функции рассматривались в [7])?

Во-вторьх, нельзя ли заменить условие (1) более сильньм так, чтобы оно влекло в.p.p. у ц.ф.э.т. с простыми вещественными нулями?

Сформулируем основные результаты работы.

Теорема 1. Для любого фиксированного $\gamma \in(0,1)$ существует и.ф.э.т. $\mathscr{L}(\lambda)$ с индикатором $h(\mathscr{L}, \theta)>0$ при произвольном $\theta \in[0,2 \pi)$ и простыми нулями $\left(\lambda_{k}\right)_{k=1}^{\infty}$, удовлетворяющая соотношению (2), но не являющаяся функиией вполне регулярного роста.

ТеОРема 2. Пусть $\mathscr{L}(\lambda)$ - и.ф.э.т. с простыми вещественными нулями $\left(\lambda_{k}\right)_{k=1}^{\infty}$, $0<\left|\lambda_{1}\right| \leqslant\left|\lambda_{2}\right| \leqslant \cdots$, причем других нулей у $\mathscr{L}$ нет. Пусть, далее, выполняется условие

$$
\exists \alpha>0: \quad \frac{1}{\left|\mathscr{L}^{\prime}\left(\lambda_{k}\right)\right|}=O\left(\left|\lambda_{k}\right|^{\alpha}\right), \quad k \rightarrow \infty .
$$

Тогда $\mathscr{L}$ имеет в.р.р. и ее индикаторная диаграмма есть отрезок мнимой оси.

Множество $S$ вещественных чисел называется относительно плотным по (лебеговой) мере $[8$, с. 112], если найдутся $\ell, \delta>0$ такие, что для любого $x \in \mathbb{R}$

$$
\operatorname{mes}(S \cap[x, x+\ell])>\delta .
$$

Пусть $S_{\gamma}$ - множество точек на прямой $\gamma(0 \in \gamma)$, полученное из $S$ поворотом вокруг начала координат на соответствующий угол. Назовем множество $S_{\gamma}$ относительно плотным по мере на прямой $\gamma$.

Теорема 3. Пусть функиия $\mathscr{L} \in L$ имеет положительный индикатор $h(\mathscr{L} ; \theta)$. Пусть также на некоторой прямой $\gamma$, проходящей через начало координат, $\mathscr{L}$ подчинена условию

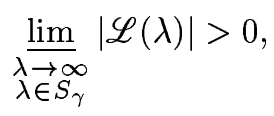




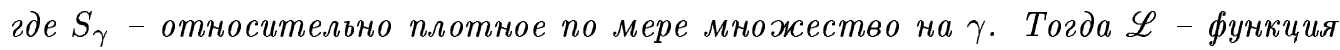
в.p.p.

Теорема 3 решает, в частности, задачу Леонтьева для функции $\mathscr{L} \in L$ с положительным нижним индикатором. Согласно, например, [9] нижний индикатор ц.ф.э.т. $\mathscr{L}$ определяется так:

$$
h_{-}(\mathscr{L}, \theta):=\sup _{E} \lim _{\substack{r \notin \infty \\ r \notin E}} \frac{\ln \left|\mathscr{L}\left(r e^{i \theta}\right)\right|}{r} .
$$

Здесь sup берется по всевозможным множествам $E$ нулевой относительной меры $\left(E^{0}\right.$-множествам), т.е. таким, что

$$
\lim _{r \rightarrow \infty} \frac{\operatorname{mes}(E \cap(0, r))}{r}=0 .
$$

В отличие от обычного индикатора нижний индикатор ц.ф.э.т. не обязан быть тригонометрически выпуклой функцией.

Отметим еще, что теорема 3 позволяет в некотором смысле уточнить ряд известных результатов о распределении показателей абсолютно представляющих систем экспонент (см., например, [5], [10]).

2. Доказательство теоремы 1. Зафиксируем $\sigma>1$ и построим такой уточненньй порядок $\rho(r) \rightarrow 1, r \rightarrow \infty$, что $1=\underline{\lim }_{r \rightarrow \infty} u_{1}(r), \sigma=\varlimsup^{\lim _{r \rightarrow \infty}} u_{1}(r)$, где $u_{1}(r)=r^{\rho(r)-1}$ (существование такой функции $\rho(r)$ анонсировано в [3]). В качестве $\rho(r)$ можно взять, например, функцию

$$
\rho(r)=1+(\ln \sigma) \cdot \frac{\sin ^{2}(\ln \ln r)}{\ln r} .
$$

Положим $u(r)=r^{\rho(r)}$ и выберем на положительном луче $R$-множество точек $\left(\mu_{k}\right)_{k=1}^{\infty}$, $\mu_{1}<\mu_{2}<\cdots$, имеющее плотность $\lim _{r \rightarrow \infty} n(r) / u(r)=\Delta>0$, где $n(r)$ - число точек $\mu_{k}$ в интервале $(0, r)$. Все точки вида $\pm \mu_{k}, \pm i \mu_{k}$ также образуют $R$-множество (при показателе $\rho(r))$, каноническая функция $\mathscr{L}$ которого представляется в виде

$$
\mathscr{L}(\lambda)=\mathscr{L}_{1}(\lambda) \cdot \mathscr{L}_{2}(\lambda), \quad \text { где } \mathscr{L}_{1}(\lambda)=\prod_{k=1}^{\infty}\left(1-\frac{\lambda^{2}}{\mu_{k}^{2}}\right), \quad \mathscr{L}_{2}(\lambda)=\prod_{k=1}^{\infty}\left(1+\frac{\lambda^{2}}{\mu_{k}^{2}}\right),
$$

и имеет индикатор (при порядке $\rho(r)) \mathscr{H}(\mathscr{L} ; \theta)=\pi \Delta(|\sin \theta|+|\cos \theta|)$. Обозначив последнее $R$-множество через $\Lambda=\left(\lambda_{k}\right)_{k=1}^{\infty},\left|\lambda_{1}\right| \leqslant\left|\lambda_{2}\right| \leqslant \cdots$, мы получим, что функция $\mathscr{L}$ имеет в.p.p. (относительно $\rho(r))$ и удовлетворяет условию

$$
\lim _{k \rightarrow \infty}\left\{\frac{1}{u\left(\left|\lambda_{k}\right|\right)} \ln \left|\mathscr{L}^{\prime}\left(\lambda_{k}\right)\right|-\mathscr{H}\left(\mathscr{L} ; \arg \lambda_{k}\right)\right\}=0
$$

Пусть $\theta \neq \pi j / 2, j=0,1,2,3$. Тогда

$$
\lim _{r \rightarrow \infty} \frac{\ln \left|\mathscr{L}\left(r e^{i \theta}\right)\right|}{u(r)}=\mathscr{H}(\mathscr{L} ; \theta) .
$$

Поскольку

$$
\frac{\ln \left|\mathscr{L}\left(r e^{i \theta}\right)\right|}{r}=\frac{\ln \left|\mathscr{L}\left(r e^{i \theta}\right)\right|}{u(r)} \cdot u_{1}(r)
$$


то

$$
\varlimsup_{r \rightarrow \infty} \frac{\ln \left|\mathscr{L}\left(r e^{i \theta}\right)\right|}{r}=\sigma \cdot \mathscr{H}(\mathscr{L} ; \theta), \quad \underline{\lim }_{r \rightarrow \infty} \frac{\ln \left|\mathscr{L}\left(r e^{i \theta}\right)\right|}{r}=\mathscr{H}(\mathscr{L} ; \theta) .
$$

Последние равенства означают, что при порядке $\rho=1$ функция $\mathscr{L}$ имеет индикатор $h(\mathscr{L}, \theta)=\sigma \cdot \mathscr{H}(\mathscr{L} ; \theta)$ и не является функцией вполне регулярного роста (относительно $\rho=1)$, ибо в противном случае из-за отсутствия у $\mathscr{L}$ нулей на луче $\arg \lambda=\theta(\theta \neq \pi j / 2$, $0 \leqslant j \leqslant 3)$ существовал бы

$$
\lim _{r \rightarrow \infty} \frac{\ln \left|\mathscr{L}\left(r e^{i \theta}\right)\right|}{r}=h(\mathscr{L} ; \theta)
$$

Имеем, наконец,

$$
\begin{aligned}
& \underline{\lim }\left\{\frac{1}{\left|\lambda_{k}\right|} \ln \left|\mathscr{L}^{\prime}\left(\lambda_{k}\right)\right|-\frac{1}{\sigma} h\left(\mathscr{L} ; \arg \lambda_{k}\right)\right\} \\
&=\frac{\lim }{k \rightarrow \infty}\left\{\frac{u_{1}\left(\left|\lambda_{k}\right|\right)}{u\left(\left|\lambda_{k}\right|\right)} \ln \left|\mathscr{L}^{\prime}\left(\lambda_{k}\right)\right|\right. \\
&\left.-u_{1}\left(\left|\lambda_{k}\right|\right) \mathscr{H}\left(\mathscr{L} ; \arg \lambda_{k}\right)+\left(u_{1}\left(\left|\lambda_{k}\right|\right)-1\right) \mathscr{H}\left(\mathscr{L} ; \arg \lambda_{k}\right)\right\} \\
& \geqslant \underset{k \rightarrow \infty}{\lim }\left\{u_{1}\left(\left|\lambda_{k}\right|\right)\left[\frac{1}{u\left(\left|\lambda_{k}\right|\right)} \ln \left|\mathscr{L}^{\prime}\left(\lambda_{k}\right)\right|-\mathscr{H}\left(\mathscr{L} ; \arg \lambda_{k}\right)\right]\right\} \\
&+\underset{k \rightarrow \infty}{\lim }\left\{\mathscr{H}\left(\mathscr{L} ; \arg \lambda_{k}\right)\left[u_{1}\left(\left|\lambda_{k}\right|\right)-1\right]\right\} \geqslant 0 .
\end{aligned}
$$

Так как индикатор $h(\mathscr{L}, \theta)$ положителен, а число $\sigma>1$ произвольно, то нужное построение завершено. Теорема доказана.

Отметим, что условие (2) естественным образом возникает при изучении $\gamma$-достаточных множеств [7] (см. также [11]), введенных Коробейником в связи с построением дискретных максимальньх множеств в некоторых классах целых функций.

3. Доказательство теоремы 2. Функция $\mathscr{L}$ может быть представлена в виде

$$
\mathscr{L}(\lambda)=e^{a \lambda+b} \prod_{k=1}^{\infty}\left(1-\frac{\lambda}{\lambda_{k}}\right) e^{\lambda / \lambda_{k}}, \quad a, b \in \mathbb{C} .
$$

Рассмотрим вспомогательную функцию

$$
\mathscr{L}_{1}(\lambda)=\left(\lambda^{2 s+1}-1\right) e^{-\lambda i \operatorname{Im} a} \cdot \mathscr{L}(\lambda), \quad \text { где } s \in \mathbb{N}, \quad 2 s \geqslant \alpha .
$$

Отметим ее свойства. Во-первых, $\mathscr{L}_{1}(\lambda)$ - ц.ф.э.т. с простыми нулями $\left(\lambda_{k}\right)_{k=1}^{\infty} \cup\left(\mu_{j}\right)_{j=0}^{2 s}$, где $\mu_{j}$ - все значения $\sqrt[2 s+1]{1}$. Далее,

$$
\mathscr{L}_{1}^{\prime}\left(\lambda_{k}\right)=\left(\lambda_{k}^{2 s+1}-1\right) e^{-\lambda_{k} i \operatorname{Im} a} \cdot \mathscr{L}^{\prime}\left(\lambda_{k}\right), \quad k=1,2, \ldots
$$

Из условия (3) в силу выбора $s$ получаем

$$
\frac{1}{\left|\mathscr{L}_{1}^{\prime}\left(\lambda_{k}\right)\right|\left|\lambda_{k}\right|}=O\left(\frac{1}{\left|\lambda_{k}\right|^{2}}\right), \quad k \rightarrow \infty
$$


Поскольку показатель сходимости

$$
\tau:=\inf \left\{\nu>0: \sum_{k=1}^{\infty} \frac{1}{\left|\lambda_{k}\right|^{\nu}}<\infty\right\}
$$

последовательности $\left(\lambda_{k}\right)_{k=1}^{\infty}$ не превосходит 1 [2, с. 27], то $\sum_{k=1}^{\infty} 1 /\left|\lambda_{k}\right|^{2}<\infty$. Отсюда

$$
\sum_{k=1}^{\infty} \frac{1}{\left|\mathscr{L}_{1}^{\prime}\left(\lambda_{k}\right)\right|\left|\lambda_{k}\right|}<\infty
$$

Кроме того,

$$
\inf \left\{\left|\mathscr{L}_{1}(i \mu)\right|: \mu \in \mathbb{R}\right\}>0 .
$$

Действительно, для всех $\mu \in \mathbb{R}$

$$
\begin{aligned}
\left|\mathscr{L}_{1}(i \mu)\right| & =\left|-1+(-1)^{s} \mu^{2 s+1} i\right| \cdot\left|e^{\mu \operatorname{Im} a+a i \mu+b}\right| \prod_{k=1}^{\infty}\left|1-i \cdot \frac{\mu}{\lambda_{k}}\right|\left|e^{i \mu / \lambda_{k}}\right| \\
& =\sqrt{1+\mu^{4 s+2}} \cdot e^{\operatorname{Re} b} \cdot \prod_{k=1}^{\infty} \sqrt{1+\left(\frac{\mu}{\lambda_{k}}\right)^{2}} \geqslant e^{\operatorname{Re} b} .
\end{aligned}
$$

Покажем, что функция $\mathscr{L}_{1}(\lambda)$ имеет в.p.p. С этой целью положим

$$
a(\lambda):=\sum_{j=0}^{2 s} \frac{\mathscr{L}_{1}(\lambda)}{\left(\lambda-\mu_{j}\right) \mathscr{L}_{1}^{\prime}\left(\mu_{j}\right)}+\sum_{k=1}^{\infty} \frac{\mathscr{L}_{1}(\lambda)}{\left(\lambda-\lambda_{k}\right) \mathscr{L}_{1}^{\prime}\left(\lambda_{k}\right)} .
$$

Нетрудно видеть, что $a(\lambda)$ - ц.ф.э.т. с индикатором $h(a ; \theta) \leqslant h\left(\mathscr{L}_{1} ; \theta\right)$ для всех $\theta \in$ $[0,2 \pi)$, причем $a\left(\mu_{j}\right)=a\left(\lambda_{k}\right)=1, j=0,1, \ldots, 2 s ; k=1,2, \ldots$ Следовательно, $b(\lambda):=$ $(a(\lambda)-1) / \mathscr{L}_{1}(\lambda)$ - ц.ф.э.т. Оценим функцию $b(\lambda)$ на мнимой оси: для всех $\mu \in \mathbb{R}$

$$
\begin{aligned}
|b(i \mu)| & \leqslant \sum_{j=0}^{2 s} \frac{1}{\left|i \mu-\mu_{j}\right|\left|\mathscr{L}_{1}^{\prime}\left(\mu_{j}\right)\right|}+\sum_{k=1}^{\infty} \frac{1}{\left|i \mu-\lambda_{k}\right|\left|\mathscr{L}_{1}^{\prime}\left(\lambda_{k}\right)\right|}+\frac{1}{\left|\mathscr{L}_{1}(i \mu)\right|} \\
& \leqslant \sum_{j=0}^{2 s} \frac{1}{\left|\operatorname{Re} \mu_{j}\right|\left|\mathscr{L}_{1}^{\prime}\left(\mu_{j}\right)\right|}+\sum_{k=1}^{\infty} \frac{1}{\left|\lambda_{k}\right|\left|\mathscr{L}_{1}^{\prime}\left(\lambda_{k}\right)\right|}+\frac{1}{\left|\mathscr{L}_{1}(i \mu)\right|} .
\end{aligned}
$$

Воспользовавшись свойствами (5) и (6) функции $\mathscr{L}_{1}$, получим, что $|b(\lambda)|$ ограничен на мнимой оси. Но тогда модуль ц.ф.э.т. $c(\lambda):=b(i \lambda)$ ограничен на вещественной оси, а значит, она принадлежит классу Картрайт и имеет в.p.p. [2, с. 323-324]. Функция $b(\lambda)$ будет также функцией в.p.p., поэтому из равенства $b(\lambda) \cdot \mathscr{L}_{1}(\lambda)=a(\lambda)-1$ заключаем, что $h(b ; \theta)+h\left(\mathscr{L}_{1} ; \theta\right) \leqslant h\left(\mathscr{L}_{1} ; \theta\right)$, или $h(b ; \theta) \leqslant 0$ для всех $\theta \in[0,2 \pi)$. Последние неравенства влекут $h(b ; \theta) \equiv 0$. Из принципа Фрагмена-Линделефа [2, с. 71] следует, что $b(\lambda) \equiv b$. Окончательно для всех $\lambda$ таких, что $\lambda \neq \mu_{j}, \lambda \neq \lambda_{k}, j=0,1, \ldots, 2 s$; $k=1,2, \ldots$, имеем представление

$$
\frac{1}{\mathscr{L}_{1}(\lambda)}=\sum_{j=0}^{2 s} \frac{1}{\mathscr{L}_{1}^{\prime}\left(\mu_{j}\right)\left(\lambda-\mu_{j}\right)}+\sum_{k=1}^{\infty} \frac{1}{\mathscr{L}_{1}^{\prime}\left(\lambda_{k}\right)\left(\lambda-\lambda_{k}\right)}-b .
$$

По теореме Крейна о разложении обратной величины целой функции [2, с. 333] $\mathscr{L}_{1}(\lambda)$ - функция в.p.p. и ее индикаторная диаграмма есть отрезок мнимой оси. По построению $\mathscr{L}_{1}(\lambda)$ этими же свойствами обладает и функция $\mathscr{L}(\lambda)$, что и завершает доказательство теоремы. 
ЗАмЕчАниЕ. В ситуации, рассмотренной в теореме 2, требование (3) более сильное, чем “условие Леонтьева” (1). В самом деле, при вьполнении (3), во-первых,

$$
h\left(\mathscr{L} ; \arg \lambda_{k}\right)=h(\mathscr{L} ; 0)=h(\mathscr{L} ; \pi)=0,
$$

а во-вторых,

$$
\varlimsup_{k \rightarrow \infty} \frac{1}{\left|\lambda_{k}\right|} \ln \frac{1}{\left|\mathscr{L}^{\prime}\left(\lambda_{k}\right)\right|} \leqslant \lim _{k \rightarrow \infty} \frac{\ln \left|\lambda_{k}\right|^{\alpha}}{\left|\lambda_{k}\right|}=0,
$$

что равносильно (1).

СлЕДСТВИЕ. Пусть $\mathscr{L}(\lambda)$ - четная (нечетная) и.ф.э.т., все отличные от нуля корни которой $\left( \pm \lambda_{k}, \lambda_{k}>0, k=1,2, \ldots\right)$ простые. Если при некотором $\alpha>0$

$$
\frac{1}{\left|\mathscr{L}^{\prime}\left(\lambda_{k}\right)\right|}=O\left(\lambda_{k}^{\alpha}\right), \quad k \rightarrow \infty
$$

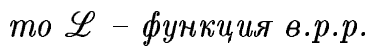

4. Вспомогательные факты. Доказательство теоремы 3. При доказательстве теоремы 3 важную роль будет играть лемма о ц.ф.э.т., сформулированная Левиньгм на конференции в Харькове в 1971 г. (для функций многих переменных) и неоднократно обобщавшаяся в различных направлениях (см., например, [12], [13]).

ЛЕмма [8]. Если $f$ - и.ф.э.т. $\sigma>0$, ограниченная на мнохестве $S$ вещественной оси, относительно плотном по мере, то

$$
\sup _{x \in \mathbb{R}}|f(x)| \leqslant[N(\ell, \delta)]^{\sigma} \sup _{x \in S}|f(x)|
$$

әде $N(\ell, \delta)$ - постоянная, зависящая лишь от указанных характеристик множества $S$ (см. введение).

Пусть $G$ - ограниченная выпуклая область в $\mathbb{C}$ с опорной функцией $h(-\theta), A(G)-$ пространство всех аналитических в $G$ функций, наделенное топологией компактной сходимости, $\Lambda=\left(\lambda_{k}\right)_{k=1}^{\infty}-$ последовательность попарно различных комплексных чисел. В соответствии с общим определением [5] последовательность $E(\Lambda)=\left(\exp \lambda_{k} z\right)_{k=1}^{\infty}$ называется абсолютно представляющей системой (АПС) в $A(G)$, если каждую функцию $x(z) \in A(G)$ можно представить в виде суммы ряда

$$
x(z)=\sum_{k=1}^{\infty} c_{k} \exp \lambda_{k} z, \quad c_{k} \in \mathbb{C}, \quad k=1,2, \ldots,
$$

абсолютно сходящегося в $A(G)$. Будем символом $[1, h(\theta)]$ обозначать множество всех целых функций, индикаторы которых (при порядке $\rho=1)$ не больше, чем $h(\theta) ;[1,0]-$ класс целых функций нулевого экспоненциального типа; $M(\Lambda ; h)$ - совокупность всех функций $\mathscr{L}(\lambda) \in[1, h(\theta)]$, для которых $\lambda_{k} \in \Lambda, k=1,2, \ldots,-$ простые нули (у $\mathscr{L}$ могут быть другие нули произвольной кратности). Приведем частные случаи (при $\rho=1)$ некоторых результатов, имеюших непосредственное отношение к данной работе. 
Teopema A [5, c. 111]. Пусть $M(\Lambda, h) \neq \varnothing$ u $\mathscr{L}(\lambda) \in M(\Lambda, h)$. Система $E(\Lambda)$ является АПС в $A(G)$ в том и только том случае, если выполняется условие: существует $D(\lambda) \in[1,0], D(\lambda) \not \equiv 0$, такая, что

$$
D(\lambda) e^{\lambda z}=\sum_{k=1}^{\infty} \frac{\mathscr{L}(\lambda)}{\lambda-\lambda_{k}} \cdot \frac{D\left(\lambda_{k}\right) e^{\lambda_{k} z}}{\mathscr{L}^{\prime}\left(\lambda_{k}\right)}, \quad z \in G, \quad \lambda \in \mathbb{C}
$$

(ряд сходится абсолютно по топологии $A(G)$ ).

Далее, для того чтобы $E(\lambda)$ была АПС в $A(G)$, необходимо, а если $\Lambda$ - совокупность всех нулей $\mathscr{L}(\lambda)$, то и достаточно, чтобы выполнялось условие: $\mathscr{L}(\lambda)$ функиия в.р.p. с индикатором $h(\theta)$ и существует $D(\lambda) \in[1,0], D(\lambda) \not \equiv 0$, такая, чmo

$$
\varlimsup_{k \rightarrow \infty}\left\{\frac{1}{\left|\lambda_{k}\right|} \ln \left|\frac{D\left(\lambda_{k}\right)}{\mathscr{L}^{\prime}\left(\lambda_{k}\right)}\right|+h\left(\arg \lambda_{k}\right)\right\} \leqslant 0 .
$$

Задача об описании в различных терминах показателей представляющих систем экспонент (или обобщенных экспонент), включая и устранение "зазора" между необходимой и достаточной частями в условии (7), получила окончательное решение в работах Абанина. Нам понадобится один из его результатов применительно к системе $E(\Lambda)$ и пространству $A(G)$.

Теорема Б $[10$, с. 809]. Для того чтобы система $E(\lambda)$ была АПС в $A(G)$, необходимо и достаточно, чтобы $\Lambda$ содержала подпоследовательность $\Lambda^{\prime} \subset \Lambda$, удовлетворяющую условию: существует и.ф.ә.т. в.р.p. $\mathscr{L}(\lambda)$ с индикатором $h(\theta)$ и простыцми нулями в точках из $\Lambda^{\prime}$, для которой совокупность всех ее нулей отличается от $\Lambda^{\prime}$ не более, чем на последовательность с нулевой плотностью и

$$
\lim _{k \rightarrow \infty, \lambda_{k} \in \Lambda^{\prime}}\left\{\frac{1}{\left|\lambda_{k}\right|} \ln \left|\mathscr{L}^{\prime}\left(\lambda_{k}\right)\right|-h\left(\arg \lambda_{k}\right)\right\}=0 .
$$

Естественным представляется вопрос о независимости условий, накладываемых на функцию $\mathscr{L}$ в теоремах А и Б. Полученные ниже в настоящей работе результаты позволяют, например, заменить в формулировках этих теорем требование полной регулярности роста $\mathscr{L}$ (равносильное условию $h_{-}(\mathscr{L} ; \theta)=h(\mathscr{L} ; \theta)$ для всех $\left.\theta \in[0,2 \pi)\right)$ на более слабое ограничение $h_{-}\left(\mathscr{L} ; \theta_{0}\right)+h_{-}\left(\mathscr{L} ; \pi+\theta_{0}\right)>0$ при некотором $\theta_{0} \in[0,2 \pi)$.

ДОКАЗАТЕЛЬСТво ТЕОРЕМЫ 3 . Введем в рассмотрение вспомогательную функцию

$$
\varphi(\lambda, z):=e^{\lambda z}-\sum_{k=1}^{\infty} \frac{\mathscr{L}(\lambda) e^{\lambda_{k} z}}{\mathscr{L}^{\prime}\left(\lambda_{k}\right)\left(\lambda-\lambda_{k}\right)}, \quad z \in G, \quad \lambda \in \mathbb{C}
$$

где $G$ - содержашая начало координат вьпуклая ограниченная область с опорной функцией, равной $h(\mathscr{L} ;-\theta)$. При фиксированном $z \in G \varphi(\lambda, z)$ является ц.ф.э.т. с индикатором $h(\varphi, \theta) \leqslant h(\mathscr{L} ; \theta)$; при фиксированном $\lambda \in \mathbb{C}$ вьполнено $\varphi(\lambda, z) \in A(G)$. Кроме того, для всех $z \in G$ выполнено $\varphi\left(\lambda_{k}, z\right)=0, k=1,2, \ldots$ Поэтому функция

$$
\psi(\lambda, z):=\frac{\varphi(\lambda, z)}{\mathscr{L}(\lambda)}=\frac{e^{\lambda z}}{\mathscr{L}(\lambda)}-\sum_{k=1}^{\infty} \frac{e^{\lambda_{k} z}}{\mathscr{L}^{\prime}\left(\lambda_{k}\right)\left(\lambda-\lambda_{k}\right)}
$$


является целой по $\lambda$ при фиксированном $z \in G$ и аналитической по $z$ в $G$ при фиксированном $\lambda \in \mathbb{C}$. Поскольку $\varphi(\cdot, z)$ и $\mathscr{L}(\lambda)$ - ц.ф.э.т., то $\psi(\cdot, z)$ - ц.ф.э.т. (при $z \in G)$. Пусть $K$ - произвольньй содержаший начало координат отрезок, лежащий в $G$, зеркальное отражение которого относительно вещественной оси перпендикулярно прямой $\gamma ; \lambda \in \gamma \backslash \Lambda$. Тогда

$$
\begin{aligned}
\max _{z \in K}|\psi(\lambda, z)| & \leqslant \frac{\max _{z \in K}\left|e^{\lambda z}\right|}{|\mathscr{L}(\lambda)|}+\sum_{k=1}^{\infty} \frac{\max _{z \in K}\left|e^{\lambda_{k} z}\right|}{\left|\mathscr{L}^{\prime}\left(\lambda_{k}\right)\right|\left|\lambda-\lambda_{k}\right|} \\
& =\frac{1}{|\mathscr{L}(\lambda)|}+\sum_{k=1}^{\infty} \frac{e^{\left|\lambda_{k}\right| h_{K}\left(\arg \lambda_{k}\right)}}{\left|\mathscr{L}^{\prime}\left(\lambda_{k}\right)\right|\left|\lambda-\lambda_{k}\right|},
\end{aligned}
$$

где $h_{K}(-\theta)$ - опорная функция $K$. Обозначим через $U$ объединение кружков

$$
U_{k}=\left\{\lambda \in \mathbb{C}:\left|\lambda-\lambda_{k}\right|<e^{-\delta\left|\lambda_{k}\right|}\right\}, \quad k=1,2, \ldots,
$$

где $\delta>0$ настолько мало, что $h_{K}(\theta)+\delta<h(\mathscr{L} ; \theta)$ при любом $\theta \in[0,2 \pi)$. Поскольку $\mathscr{L}$ - ц.ф.э.т., то для любого $\delta>0$ выполнено $\sum_{k=1}^{\infty} e^{-\delta\left|\lambda_{k}\right|}<\infty$, а множество $\left\{r>0: \lambda=r e^{i \theta} \in \gamma \cap U\right\}$ либо пусто, либо является $E^{0}$-множеством. Тогда $S_{1, \gamma}:=$ $S_{\gamma} \backslash U$ относительно плотно по мере на $\gamma$, и в силу (4)

$$
\underset{\substack{\lim \\ \lambda \in S_{1, \gamma}}}{ }|\mathscr{L}(\lambda)|>0
$$

Отсюда, из условия (1) и оценки

$$
\max _{z \in K}|\psi(\lambda, z)| \leqslant \frac{1}{|\mathscr{L}(\lambda)|}+\sum_{k=1}^{\infty} \frac{e^{\left|\lambda_{k}\right|\left[h_{K}\left(\arg \lambda_{k}\right)+\delta\right]}}{\left|\mathscr{L}^{\prime}\left(\lambda_{k}\right)\right|} \quad \forall \lambda \in \gamma \backslash U
$$

получаем, что при любом фиксированном $z \in K$ модуль $\psi(\lambda, z)$ ограничен на множестве $S_{1, \gamma}$. При соответствуюшем выборе $\theta_{0} \in[0,2 \pi)$ модуль ц.ф.э.т. (по $\left.\lambda\right) \psi\left(\lambda e^{i \theta_{0}}, z\right)$ ограничен на некотором относительно плотном по мере множестве $S_{1} \subset \mathbb{R}$. Если бы ее экспоненциальньй тип был больше нуля, то по лемме Левина модуль этой функции был бы ограничен на вешественной оси. Тогда указанная функция принадлежала бы классу Картрайт и имела в.p.p. Воспользовавшись тогда для функции в.p.p. $\psi(\cdot, z)$ с индикатором $h_{z}(\psi, \theta)$ представлением (при $z \in K$ )

$$
\psi(\lambda, z) \cdot \mathscr{L}(\lambda)=e^{\lambda z}-\sum_{k=1}^{\infty} \frac{\mathscr{L}(\lambda) e^{\lambda_{k} z}}{\mathscr{L}^{\prime}\left(\lambda_{k}\right)\left(\lambda-\lambda_{k}\right)}, \quad \lambda \in \mathbb{C}
$$

мы бы получили

$$
h_{z}(\psi, \theta)+h(\mathscr{L}, \theta) \leqslant h(\mathscr{L}, \theta), \quad \theta \in[0,2 \pi),
$$

откуда $h_{z}(\psi, \theta) \equiv 0$, что по предположению невозможно. Таким образом, при любом $z \in K \psi(\cdot, z) \in[1,0]$. Применив к целой функции $\psi\left(\lambda e^{i \theta_{0}}, z\right) \cdot e^{-i \lambda}$ экспоненциального типа 1 лемму Левина, найдем, что $|\psi(\cdot, z)|$ ограничен на прямой $\gamma$. Следовательно, $\psi(\cdot, z) \equiv c_{z}$ при любом $z \in K$. Зафиксируем $z \in K$ и вернемся к оценке

$$
|\psi(\lambda, z)| \leqslant \frac{1}{|\mathscr{L}(\lambda)|}+\left|\sum_{k=1}^{\infty} \frac{e^{\lambda_{k} z}}{\mathscr{L}^{\prime}\left(\lambda_{k}\right)\left(\lambda-\lambda_{k}\right)}\right|, \quad \lambda \in \gamma \backslash \Lambda .
$$


Второе слагаемоев правой части последнего неравенства стремится к нулю при $\lambda \rightarrow \infty$, $\lambda \notin U$. Выберем согласно теореме Бернштейна (см., например [2, с. 99]) на одном из двух лучей $\gamma\left(\theta_{0}\right)$, составляющих прямую $\gamma$, последовательность точек

$$
\mu_{j}=r_{j} e^{i \theta_{0}}, \quad \mu_{j} \rightarrow \infty, \quad j \rightarrow \infty
$$

так, чтобы $r_{j} \notin E$ и

$$
\left|\mathscr{L}\left(\mu_{j}\right)\right|>\exp \left(\frac{1}{2} h\left(\mathscr{L} ; \theta_{0}\right) r_{j}\right), \quad j=1,2, \ldots
$$

Получим

$$
\frac{1}{\mathscr{L}\left(\mu_{j}\right)} \rightarrow 0, \quad j \rightarrow \infty, \quad \sum_{k=1}^{\infty} \frac{e^{\lambda_{k} z}}{\mathscr{L}^{\prime}\left(\lambda_{k}\right)\left(\mu_{j}-\lambda_{k}\right)} \rightarrow 0, \quad j \rightarrow \infty,
$$

откуда $\psi\left(\mu_{j}, z\right) \rightarrow 0, j \rightarrow \infty$. Поэтому $\psi(\lambda, z)=0$ для всех $\lambda \in \mathbb{C}$ и $z \in K$. По теореме единственности $\psi(\lambda, z)=0$ для всех $\lambda \in \mathbb{C}$ и $z \in G$. Таким образом, имеет место равенство

$$
e^{\lambda z}=\sum_{k=1}^{\infty} \frac{\mathscr{L}(\lambda) e^{\lambda_{k} z}}{\mathscr{L}^{\prime}\left(\lambda_{k}\right)\left(\lambda-\lambda_{k}\right)}, \quad \lambda \in \mathbb{C}, \quad z \in G .
$$

Согласно теореме А $E(\Lambda)$ - $\mathrm{A \Pi C}$ в $A(G)$, а $\mathscr{L}(\lambda)$ - функция в.р.р. Теорема доказана.

СЛЕДСТВИЕ 1. Пусть мнохсество внутренних точек индикаторной диаграммы функиии $\mathscr{L} \in L$ непусто, и при некотором $\theta_{0} \in[0,2 \pi)$ выполнена оченка

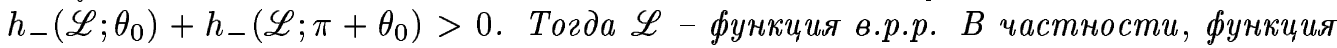
класса $L$ с тригонометрически выпуклым или положстельным нижним индикатором имеет в.р.p.

ДоКАЗАТЕЛЬСТВО. Без ограничения общности можно считать, что $h_{-}\left(\mathscr{L} ; \theta_{0}\right)=$ $h_{-}\left(\mathscr{L} ; \pi+\theta_{0}\right)>0$. В самом деле, общий случай легко свести к указанному, рассмотрев вместо функции $\mathscr{L}$ функцию $\mathscr{L}_{1}(\lambda)=\mathscr{L}(\lambda) \cdot e^{a \lambda}$, где

$$
\begin{aligned}
|a| & =\frac{1}{2}\left|h_{-}\left(\mathscr{L} ; \theta_{0}\right)-h_{-}\left(\mathscr{L} ; \pi+\theta_{0}\right)\right|, \\
\arg a & = \begin{cases}-\theta_{0}, & \text { если } h_{-}\left(\mathscr{L} ; \theta_{0}\right) \leqslant 0, \\
\pi-\theta_{0}, & \text { если } h_{-}\left(\mathscr{L} ; \pi+\theta_{0}\right) \leqslant 0 .\end{cases}
\end{aligned}
$$

Нетрудно проверить, что $\mathscr{L}_{1} \in L_{\text {и }}$

$$
h_{-}\left(\mathscr{L}_{1} ; \theta_{0}\right)=h_{-}\left(\mathscr{L}_{1} ; \pi+\theta_{0}\right)=\frac{1}{2}\left(h_{-}\left(\mathscr{L} ; \theta_{0}\right)+h_{-}\left(\mathscr{L} ; \pi+\theta_{0}\right)\right)>0
$$

Кроме того, при подходящем выборе $b \in \mathbb{C}$ функция $\mathscr{L}_{2}(\lambda)=\mathscr{L}_{1}(\lambda) e^{b \lambda}$, наряду с отмеченньми свойствами, обладает положительным индикатором. Считая таковой функцию $\mathscr{L}$, найдем $E^{0}$-множество $E_{1}$ так, чтобы

$$
\underset{r \notin \infty}{\lim \underset{r \notin E_{1}}{\longrightarrow}} \frac{\ln \left|\mathscr{L}\left(r e^{i \theta_{0}}\right)\right|}{r}>\frac{1}{2} h_{-}\left(\mathscr{L} ; \theta_{0}\right) .
$$


Тогда для всех $r \geqslant r_{1}, r \notin E_{1}$ вьполнена оценка

откуда

$$
\left|\mathscr{L}\left(r e^{i \theta_{0}}\right)\right|>\exp \frac{1}{2} h_{-}\left(\mathscr{L} ; \theta_{0}\right) r
$$

$$
\lim _{\substack{r \rightarrow \infty \\ r \notin E_{1}}}\left|\mathscr{L}\left(r e^{i \theta_{0}}\right)\right|=+\infty .
$$

Аналогично, найдется $E^{0}$-множество $E_{2}$ со свойством

$$
\lim _{\substack{r \rightarrow \infty \\ r \notin E_{2}}}\left|\mathscr{L}\left(r e^{i\left(\theta_{0}+\pi\right)}\right)\right|=+\infty .
$$

Множество $S:=\mathbb{R} \backslash\left\{E_{1} \cup\left(-E_{2}\right)\right\}$ относительно плотно по мере, и на прямой $\gamma:=\{0\} \cup$ $\left\{\lambda \in \mathbb{C}: \arg \lambda=\theta_{0}\right\} \cup\left\{\lambda \in \mathbb{C}: \arg \lambda=\pi+\theta_{0}\right\}$

$$
\lim _{\substack{\lambda \rightarrow \infty \\ \lambda \in S_{\gamma}}}|\mathscr{L}(\lambda)|=+\infty
$$

По теореме $3 \mathscr{L}$ имеет в.p.p.

СледСТВИЕ 2. Пусть $\mathscr{L}$ - четная (нечетная) функиия класса $L$ с отличной от отрезка индикаторной диаграммой. Eсли $h_{-}\left(\mathscr{L} ; \theta_{0}\right)>0$ при некотором $\theta_{0} \in[0,2 \pi)$, то $\mathscr{L}$ имеет в.р.p. В частности, четная (нечетная) функиия $\mathscr{L} \in L$ с положительным индикатором, имеющая в.р.р. на каком-либо луче, есть функиия в.р.р.

СледствИЕ 3. Четная (нечетная) функиия класса L с положительным индикатором, не имеющая нулей в каком-либо угле раствора $\pi / 2$, является функиией в.p.p.

ДокАЗАТЕЛЬСТВо. Пусть угол

$$
\Gamma\left(\theta_{0}\right):=\left\{\lambda \in \mathbb{C}:\left|\arg \lambda-\theta_{0}\right|<\frac{\pi}{4}\right\}
$$

не содержит нулей функции $\mathscr{L}$. Не умаляя общности рассуждений, будем предполагать, что

$$
\mathscr{L}(\lambda)=\prod_{k=1}^{\infty}\left(1-\frac{\lambda^{2}}{\lambda_{k}^{2}}\right) .
$$

В силу четности $\mathscr{L}$ все ее нули лежат вне множества $\Gamma\left(\theta_{0}\right) \cup\left(-\Gamma\left(\theta_{0}\right)\right)$, поэтому

$$
\frac{\pi}{4} \leqslant\left|\theta_{0}-\arg \lambda_{k}\right| \leqslant \frac{3 \pi}{4}, \quad k=1,2, \ldots
$$

Оценим снизу $|\mathscr{L}(\lambda)|$ на луче $\gamma\left(\theta_{0}\right):=\left\{\lambda \in \mathbb{C}: \arg \lambda=\theta_{0}\right\}$. Имеем для произвольного $\lambda \in \gamma\left(\theta_{0}\right)$

$$
\begin{aligned}
|\mathscr{L}(\lambda)|=\prod_{k=1}^{\infty}\left|1-\frac{\lambda^{2}}{\lambda_{k}^{2}}\right| & =\prod_{k=1}^{\infty} \sqrt{1-2\left|\frac{\lambda}{\lambda_{k}}\right|^{2} \cos 2\left(\theta_{0}-\arg \lambda_{k}\right)+\left|\frac{\lambda}{\lambda_{k}}\right|^{4}} \\
& >\prod_{k=1}^{\infty} \sqrt{1-2\left|\frac{\lambda}{\lambda_{k}}\right|^{2} \cos 2\left|\theta_{0}-\arg \lambda_{k}\right| \geqslant 1 .}
\end{aligned}
$$

Следовательно, и на прямой $\gamma:=\{0\} \cup \gamma\left(\theta_{0}\right) \cup\left(-\gamma\left(\theta_{0}\right)\right)$ вьполнена оценка $|\mathscr{L}(\lambda)| \geqslant 1$. Доказательство завершается применением теоремы 3. 
5. Теоремы типа Левинсона и представляющие системы экспонент. Применим следствие 3 теоремы 3 к одной конкретной ситуации.

ТЕорема 4. Пусть числа $0<\nu_{n}, \mu_{n}<\infty$ таковы, что

$$
\begin{aligned}
& 0<\underline{\lim } \frac{n}{\nu_{n}} \leqslant \varlimsup_{n \rightarrow \infty} \frac{n}{\nu_{n}}=D_{1}<\infty, \\
& 0<\underset{n \rightarrow \infty}{\underline{\lim } \frac{n}{\mu_{n}}} \leqslant \varlimsup_{n \rightarrow \infty} \frac{n}{\mu_{n}}=D_{2}<\infty,
\end{aligned}
$$

и при этом

$$
\mathscr{L}(\lambda):=\prod_{n=1}^{\infty}\left(1-\frac{\lambda^{2}}{\nu_{n}^{2}}\right) \cdot \prod_{n=1}^{\infty}\left(1+\frac{\lambda^{2}}{\mu_{n}^{2}}\right) \in L .
$$

Пусть, далее, $\Phi(\lambda)$ - и.ф.э.т. такая, что ее тип $\sigma_{\Phi}<\pi \sqrt{D_{1}^{2}+D_{2}^{2}}$, причем

$$
\begin{aligned}
& \alpha_{1}=\max \left[\varlimsup_{n \rightarrow \infty} \frac{\ln \left|\Phi\left(\nu_{n}\right)\right|}{\nu_{n}}, \varlimsup_{n \rightarrow \infty} \frac{\ln \left|\Phi\left(-\nu_{n}\right)\right|}{\nu_{n}}\right]<\pi D_{2}, \\
& \alpha_{2}=\max \left[\varlimsup_{n \rightarrow \infty} \frac{\ln \left|\Phi\left(i \mu_{n}\right)\right|}{\mu_{n}}, \varlimsup_{n \rightarrow \infty} \frac{\ln \left|\Phi\left(-i \mu_{n}\right)\right|}{\mu_{n}}\right]<\pi D_{1} .
\end{aligned}
$$

Tогда

$$
\begin{aligned}
& \text { 1) } \sup \left\{\frac{h(\Phi ; \theta)}{h(\mathscr{L} ; \theta)}: \theta \in[0,2 \pi)\right\}=\max \left\{\frac{\alpha_{1}}{\pi D_{2}}, \frac{\alpha_{2}}{\pi D_{1}}\right\} \\
& \text { 2) } \sup _{\lambda \in \mathbb{C}}|\Phi(\lambda)|=\max \left\{\sup _{n \geqslant 1}\left|\Phi\left( \pm \nu_{n}\right)\right|, \sup _{n \geqslant 1}\left|\Phi\left( \pm i \mu_{n}\right)\right|\right\} .
\end{aligned}
$$

ДокАЗАтЕльство. Четная ц.ф.э.т. $\mathscr{L}$ принадлежит классу $L$, не имеет нулей, например, в угле $\{\lambda \in \mathbb{C}: 0<\arg \lambda<\pi / 2\}$, и ее индикатор положителен. В самом деле, всякая ц.ф.э.т. $F \not \equiv 0$ удовлетворяет неравенству [2, с. 245]

$$
\varliminf_{r \rightarrow \infty} \frac{\nu_{F}(r)}{r} \leqslant \frac{1}{2 \pi}\left[h\left(F ; \frac{\pi}{2}\right)+h\left(F ;-\frac{\pi}{2}\right)\right],
$$

где $\nu_{F}(r)$ - число нулей $F$ в круге $\{\lambda \in \mathbb{C}:|\lambda-r / 2| \leqslant r / 2\}$. Поэтому

$$
h\left(\mathscr{L} ; \frac{\pi}{2}\right) \geqslant \pi \lim _{n \rightarrow \infty} \frac{n}{\nu_{n}}>0 .
$$

Аналогично, $h(\mathscr{L} ; 0)>0$. Следовательно, $h(\mathscr{L} ; \theta)>0$ для всех $\theta$. По следствию 3 теоремы $3 \mathscr{L}$ - функция в.p.p. Но тогда последовательности $\left(\nu_{n}\right)_{n=1}^{\infty}$ и $\left(\mu_{n}\right)_{n=1}^{\infty}$ имеют плотности $D_{1}$ и $D_{2}$ соответственно. Остается применить теорему 10 из [7].

Установленное утверждение уточняет часть цитированного вьше результата Коробейника [7] (теорема 10 при $\gamma=1$ ). Отметим еще вытекающее из теоремы 4 обобщение известной теоремы Левинсона (см., например, [2, с. 267]).

СЛЕДСТВИЕ. Пусть числа $\nu_{n} u \mu_{n}$ такие же, как и в теореме 4, и пусть $\Phi(\lambda)$ - и.ф.э.т. такая, что ее тип $\sigma_{\Phi}<\pi \sqrt{D_{1}^{2}+D_{2}^{2}}$, ограниченная на мнохестве $\left\{ \pm \nu_{n}, \pm i \mu_{n}\right\}$. Тогда $\Phi(\lambda) \equiv$ const.

Для формулировки следующего результата введем некоторые обозначения. Символом $v(\mathscr{L})$ обозначим совокупность всех нулей функции $\mathscr{L}$. Пусть $M(\Lambda ; h)$ - класс функций, определенный в п. $4 ; \widetilde{M}(\Lambda ; h):=\{\mathscr{L} \in M(\Lambda ; h): v(\mathscr{L}) \backslash \Lambda$ - множество не более чем нулевой плотности\}. 
ТЕОрема 5. Пусть $G$ - выпуклая ограниченная область с опорной функиией $h(-\theta), \widetilde{M}(\Lambda ; h) \neq \varnothing, \mathscr{L} \in \widetilde{M}(\Lambda ; h)$. Следующие условия равносильны:

1) индикатор функиии $\mathscr{L}$ совпадает с $h(\theta)$, ее нижний индикатор удовлетворяет при некотором $\theta_{0} \in[0,2 \pi)$ неравенству $h_{-}\left(\mathscr{L} ; \theta_{0}\right)+h_{-}\left(\mathscr{L} ; \pi+\theta_{0}\right)>0$, и выполняется неравенство (7);

2) $\mathscr{L}$ - функиия в.р.p. с индикатором $h(\theta)$, причем имеет место (7);

3) $E(\Lambda)-\mathrm{A \Pi C}$ в $A(G)$.

ДокАЗАТЕЛЬСтво. 2) $\Rightarrow 1$ ). Пусть вьполнено условие 2$)$. Тогда для всех $\theta$ выполнено $h_{-}(\mathscr{L} ; \theta)=h(\mathscr{L} ; \theta)=h(\theta)$. Из неравенства $\inf _{\theta}\{h(\theta)+h(\pi+\theta)\}>0$ заключаем, что имеет место условие 1$)$.

$1) \Rightarrow 3)$. Подберем, как и при доказательстве следствия 1 теоремы 3 , число $c \in \mathbb{C}$ так, чтобы функция $\mathscr{L}_{1}(\lambda)=e^{c \lambda} \cdot \mathscr{L}(\lambda)$ удовлетворяла условиям: $h\left(\mathscr{L}_{1} ; \theta\right)>0$ при всех $\theta$, $h_{-}\left(\mathscr{L}_{1} ; \theta_{0}\right)=h_{-}\left(\mathscr{L}_{1} ; \pi+\theta_{0}\right)>0$. При этом, для $\mathscr{L}_{1}$ вьполняется неравенство $(7)$. Пусть $\Lambda^{\prime}:=v(\mathscr{L}) \backslash \Lambda \neq \varnothing$. Построим по множеству $\pm \Lambda^{\prime}$ каноническое произведение Вейерштрасса $a(\lambda)$. Из определения класса $\widetilde{M}(\Lambda ; h)$ следует, что $a(\lambda) \in[1,0], a\left(\lambda_{k}\right) \neq 0$, $k=1,2, \ldots$. Тогда $d(\lambda):=D(\lambda) a(\lambda) \in[1,0]$ и

$$
\varlimsup_{k \rightarrow \infty}\left\{\frac{1}{\left|\lambda_{k}\right|} \ln \left|\frac{d\left(\lambda_{k}\right)}{\mathscr{L}_{1}^{\prime}\left(\lambda_{k}\right)}\right|+h\left(\mathscr{L}_{1} ; \arg \lambda_{k}\right)\right\} \leqslant 0 .
$$

Рассмотрим, следуя доказательству теоремы 3 , вспомогательную функцию

$$
\varphi_{d}(\lambda, w):=d(\lambda) e^{\lambda w}-\sum_{k=1}^{\infty} \frac{\mathscr{L}_{1}(\lambda) d\left(\lambda_{k}\right) e^{\lambda_{k} w}}{\mathscr{L}_{1}^{\prime}\left(\lambda_{k}\right)\left(\lambda-\lambda_{k}\right)}, \quad \lambda \in \mathbb{C}, \quad w \in G_{1}
$$

$\left(G_{1}\right.$ - вьпуклая область с опорной функцией $\left.h\left(\mathscr{L}_{1} ;-\theta\right)>0\right)$. Она обладает всеми свойствами функции $\varphi$ и, кроме того, $\varphi_{d}(\mu, w)=0$ для всех $\mu \in v(\mathscr{L})$ и $w \in G_{1}$. Поэтому,

$$
\psi_{d}(\lambda, w):=\frac{\varphi_{d}(\lambda, w)}{\mathscr{L}_{1}(\lambda)}=\frac{d(\lambda) e^{\lambda w}}{\mathscr{L}_{1}(\lambda)}-\sum_{k=1}^{\infty} \frac{d\left(\lambda_{k}\right) e^{\lambda_{k} w}}{\mathscr{L}_{1}^{\prime}\left(\lambda_{k}\right)\left(\lambda-\lambda_{k}\right)}
$$

- ц.ф.э.т. по $\lambda$ (при фиксированном $\left.w \in G_{1}\right)$. Используя условия $h_{-}\left(\mathscr{L}_{1} ; \theta\right)>0$, $d(\lambda) \in[1,0]$ так же, как при доказательстве теоремы 3 , устанавливаем, что для всех $w \in K_{1}\left|\psi_{d}(\lambda, w)\right|$ ограничен на множестве $S_{\gamma}$. Здесь $\gamma=\{0\} \cup \gamma\left(\theta_{0}\right) \cup \gamma\left(\pi+\theta_{0}\right), S_{\gamma}$ - относительно плотное по мере множество на $\gamma, K_{1}$ - некоторый отрезок, лежащий в области $G_{1}, 0 \in K_{1} \perp \gamma\left(-\theta_{0}\right)$.

Отсюда известным уже способом выводим, что $\psi_{d}(\lambda, w) \equiv 0, \lambda \in \mathbb{C}, w \in G_{1}$, и получаем равенство

$$
d(\lambda) e^{\lambda w}=\sum_{k=1}^{\infty} \frac{\mathscr{L}_{1}(\lambda) d\left(\lambda_{k}\right) e^{\lambda_{k} w}}{\mathscr{L}_{1}^{\prime}\left(\lambda_{k}\right)\left(\lambda-\lambda_{k}\right)}, \quad \lambda \in \mathbb{C}, \quad w \in G_{1} .
$$

Сделав замену $z=w-c$, приходим к соотношению

$$
d(\lambda) e^{\lambda z}=\sum_{k=1}^{\infty} \frac{\mathscr{L}(\lambda) d\left(\lambda_{k}\right) e^{\lambda_{k} z}}{\mathscr{L}^{\prime}\left(\lambda_{k}\right)\left(\lambda-\lambda_{k}\right)}, \quad \lambda \in \mathbb{C}, \quad z \in G,
$$

равносильному по первой части теоремы А условию 3). Справедливость импликации $3) \Rightarrow 2$ ) следует непосредственно из второй части теоремы А.

Таким образом, 1) $\Leftrightarrow 2) \Leftrightarrow 3)$. Теорема доказана.

Теорема 5 служит дополнением к теореме А. В то же время, она дает возможность в некотором смысле усилить теорему Б. Именно, справедливо следующее утверждение. 
ТЕорема 6. Для того чтобы система $E(\Lambda)$ была АПС в $A(G)$, необходимо и достаточно, чтобы $\Lambda$ содержала подпоследовательность $\Lambda^{\prime} \subset \Lambda$, удовлетворяющую условию: существует функиия $\mathscr{L} \in \widetilde{M}\left(\Lambda^{\prime}, h\right)$ с индикатором $h(\theta)$, для которой при некотором $\theta_{0} \in[0,2 \pi)$ выполнено $h_{-}\left(\mathscr{L} ; \theta_{0}\right)+h_{-}\left(\mathscr{L} ; \pi+\theta_{0}\right)>0 u$

$$
\lim _{k \rightarrow \infty, \lambda_{k} \in \Lambda^{\prime}}\left\{\frac{1}{\left|\lambda_{k}\right|} \ln \left|\mathscr{L}^{\prime}\left(\lambda_{k}\right)\right|-h\left(\arg \lambda_{k}\right)\right\}=0 .
$$

ДоКАЗАТЕЛЬСТВо. Необходимость легко вьводится на основании "необходимой" части теоремы Б точно так же, как импликация 2) $\Rightarrow 1$ ) при доказательстве теоремы 5 .

Достаточность. Пусть функция $\mathscr{L}$ удовлетворяет всем требованиям, присутствующим в условии теоремы 6 . Тогда для $\Lambda^{\prime}$ имеет место условие 1 ) теоремы 5, причем неравенство (7) вьполняется с $D(\lambda) \equiv 1$. Следовательно, последовательность $E\left(\Lambda^{\prime}\right)$ образует по теореме $5 \mathrm{A \Pi C}$ в $A(G)$. Подавно, $E(\Lambda)-\mathrm{A \Pi C}$ в $A(G)$. Достаточность, а вместе с ней и теорема, доказана.

Автор выражает благодарность профессору В. А. Осколкову и профессору А. В. Абанину за полезное обсуждение результатов статьи.

\section{СПИСОК ЦИТИРОВАННОЙ ЛИТЕРАТУРЫ}

[1] Леонтьев А.Ф. Об условиях разрешимости аналитических функций в ряды Дирихле // Изв. АН СССР. Сер. матем. 1972. Т. 36. №6. С. 1282-1295.

[2] Левин Б.Я. Распределение корней целых функций. М.: Гостехиздат, 1956.

[3] Братищев А. В. К одной задаче А. Ф. Леонтьева // Докл. АН СССР. 1983. Т. 270. № 2. C. $265-267$.

[4] Мельник Ю.И. О представлении регулярных функций рядами типа рядов Дирихле // Исследование по теории приближения функций и их приложения. Киев, 1978. С. 132-141.

[5] Коробейник Ю. Ф. Представляющие системы // УМН. 1981. Т. 36. № 1. С. 73-126.

[6] Братищев А. В. Базисы Кете, целые функции и их приложения // Дисс. ... д.ф.-м.н. Екатеринбург, 1995.

[7] Коробейник Ю.Ф. Максимальные и $\gamma$-достаточные множества. Приложения к цельм функциям. 2 // Теория функций, функциональный анализ и их приложения. Т. 55 . Харьков, 1991. С. 23-34.

[8] Левин Б.Я. Почти периодические функции с ограниченным спектром // Актуальные вопросы математического анализа. Ростов-на-Дону: Изд-во РГУ, 1978. С. 112-124.

[9] Азарин В.С. Пример целой функции с заданным индикатором и нижним индикатором // Матем. сб. 1972. Т. 89 (131). № 4. С. 541-557.

[10] Абанин А. В. Геометрические критерии представления аналитических функций рядами обобщенных экспонент // Докл. РАН. 1992. Т. 323. № 5. С. 807-810.

[11] Шерстюков В. Б. К вопросу о $\gamma$-достаточных множествах // Сиб. матем. ж. 2000. Т. 41. № 4. C. $935-943$.

[12] Кацнельсон В. Э. Эквивалентные нормы в пространствах целых функций экспоненциального типа // Матем. сб. 1973. Т. 92. № 2. С. 543-547.

[13] Логвиненко В. Н. Об одном многомерном обобщении теоремы М. Картрайт // Докл. АН CCCP. 1974. T. 219. № 3. C. 546-549.

Московский государственный

Поступило

инженерно-физический институт 08.02 .2002 